\title{
Compétition pour la lumière et morphologie du trèfle blanc (Trifolium repens L.) : émission des feuilles et des ramifications
}

\author{
J.C. Simon ${ }^{1}$, F. Gastal ${ }^{2}$ et G. Lemaire ${ }^{2}$ \\ 1 INRA, Station d'agronomie, 29000 Quimper; \\ 2 INRA, Station d'écophysiologie des plantes fourragères, 86600 Lusignan, France
}

(reçu le 11-7-1988, accepté le 10-1-1989)

\begin{abstract}
Résumé - La morphogénèse du trèfle blanc a été étudiée sur des couverts de trèfle blanc pur ou associé (avec un ray-grass anglais ou une fétuque élevée), en phase d'implantation ou en culture bien implantée. Différentes études, conduites à Quimper (climat doux et humide) et à Lusignan (climat chaud et sec), montrent qu'au cours d'une pousse ou repousse deux phases se succèdent:

- quand l'indice foliaire du couvert est inférieur à 3, c'est-à-dire en l'absence de compétition pour la lumière, la vitesse d'émission des feuilles et des nouveaux points végétatifs est essentiellement liée à la température;

- quand l'indice foliaire dépasse 3, le rythme d'émission des feuilles diminue et la ramification s'arrête. Dans le même temps, l'élongation des extrémités des apex de stolons s'accélère. L'indice foliaire 3 correspond à une quasi extinction du rayonnement solaire transmis à la base des plantes, au niveau des sites d'émission des futurs organes.

En l'absence de compétition pour la lumière, sur plantes ou stolons isolés, les vitesses d'apparition des feuilles et des ramifications sont en relation avec la température. Ces résultats confirment l'effet direct de la lumière sur la morphologie, déjà mis en évidence sur graminées fourragères tempérées.
\end{abstract}

morphogénèse - stolons - élongation

Summary - White clover morphogenesis and light competition: leaves and growing points emergence. The relationship between white clover morphogenesis and light competition has been studied during sward establishment, and on established swards of white clover in pure-stands or mixtures. Different experiments conducted in Quimper (fresh and wet weather) and in Lusignan (hot and dry weather), show 2 phasis during a regrowth (or during sward establishement) according to morphological phenomenons:

- when Leaf Area Index (LAI) is smaller than 3, leaves and growing points emergence rates are mainly related to temperature;

- when $L A l$ reaches a value 3 , leaf emergence decreases rapidly and branching terminates. In the same time, stolon elongation increases quickly. This LAI 3 corresponds with the almost complete extinction of light at the basis of plants.

On isolated plants or stolons, without competition for light, leaf appearance rate and branch emission rate are mostIy in relationship with temperature. These results confirm the direct effect of light on morphogenesis previously demonstrated for temperate grasses.

morphogenesis - stolon - elongation

\section{Introduction}

La productivité d'un couvert végétal dépend du rayonnement intercepté par son appareil foliaire (Monteith, 1977; Gallagher et Biscoe, 1978; Varlet-Grancher et Bonhomme, 1982). Ce rayonnement intercepté dépend essentiellement de l'indice foliaire (Brown et Blaser, 1968; Bonhomme et Varlet-Grancher, 1977 et 1979; Gosse et al.
1984) et du rayonnement incident. Pour comprendre l'élaboration de la biomasse et les variations de composition des mélanges graminée trèfle blanc, il faut connaître les mécanismes de mise en place de l'appareil foliaire des 2 espèces.

L'indice foliaire du trèfle blanc dépend de 3 paramètres caractérisant la morphologie du cou- 
vert: le nombre de points végétatifs, le nombre de feuilles par point végétatif, la surface d'une feuille. Dans cet article, nous proposons d'étudier la mise en place des feuilles et des points végétatifs: vitesse d'apparition des organes nouveaux, incidences des phénomènes de compétition pour la lumière sur la dynamique d'émission de ces organes.

\section{Méthodologie}

\section{La plante}

C'est la variété de trèfle blanc Grasslands Huia, de type hollandicum, qui a servi de support à nos études. Ce trèfle a été associé, ou non, à une graminée fourragère, un ray-grass anglais (Vigor), dans les essais de Quimper ou une fétuque élevée (Clarine) dans les essais de Lusignan.

\section{Définitions: feuilles et points végétatifs}

Pour un point végétatif donné, une nouvelle feuille est comptabilisée quand la nervure centrale de cette dernière est visible sur au moins l'un de ses folioles. Un point végétatif est considéré comme apparu quand il présente au moins une feuille décrite comme ci-dessus.

Si les apparitions d'organes ne posent guère de problèmes en phase d'implantation de la culture, (plante jeune présentant un nombre limité de feuilles et de ramifications), il n'en est pas de même en couvert bien implanté où la morphologie du trèfle blanc est très complexe. On note en particulier une grande variabilité morphologique des points végétatifs. Aussi, afin de mieux caractériser cette variabilité et afin de préciser si celle-ci a des incidences sur le devenir des différents points, nous avons été amenés à en définir arbitrairement 3 types principaux.

Ces trois types sont:

- les apex principaux (a), points végétatifs très vigoureux où l'élongation du stolon est rapide;

- les apex secondaires (b), extrémités de stolons où la croissance récente est faible: moins de 2 centimètres;

- les points axillaires (c), situés à la base des feuilles. Ces ébauches de ramification du stolon ont une élongation nulle; elles ne produisent que des feuilles disposées en rosette.

Quand les conditions d'environnement sont favorables, les types "b" et "c" ne sont que des étapes transitoires conduisant in fine au type "a". De ce fait, à l"exception de ce dernier type, on peut parfois observer un changement de type du point végétatif entre le début et la fin d'une expérimentation (passage du type "b" au type "a", par exemple). Le type indiqué dans les expérimentations suivantes correspond à l'état du point végétatif au moment du baguage.

\section{Prélèvements et baguages}

Afin de suivre les cinétiques d'apparition des feuilles et d'émission de nouveaux points végétatifs, 2 méthodes ont été employées selon l'âge du couvert végétal :

- en phase d'implantation, sur des plantes jeunes facilement individualisables, le suivi est fait sur des plantes en place bien repérées. La cadence des observations est hebdomadaire ou bihebdomadaire;

- en couvert bien implanté, sur des plantes qui ont développé un puissant réseau de stolons, les observations sont faites à la même cadence que ci-dessus, sur 20 points végétatifs bagués.

\section{Les traitements}

La production d'organes nouveaux (feuilles et points végétatifs) a été analysée dans 2 situations distinctes:

- en l'absence de compétition pour la lumière, sur des plantes isolées ou sur des stolons maintenus à la lumière en rasant le couvert à la périphérie;

- en conditions de compétition intra- ou interspécifique (avec ou sans graminée associée); en couvert bien implanté, les suivis ont été menés sur des cultures mises en place depuis 2 ans.

Dans les 2 situations, ce sont les mêmes parcelles qui servent de support aux études (avec ou sans compétition), sur une même période de croissance, donc avec un état initial identique.

Les expérimentations ont été mises en place à Quimper (Bretagne occidentale, au climat frais et humide) et à Lusignan (Poitou, au climat plus chaud et $\mathrm{sec}$ ). Les expérimentations sur la phase d'implantation n'ont été développées qu'à Quimper.

\section{Expression des résultats}

Les cinétiques de production d'organes nouveaux sont exprimées en fonction du temps. Les barres verticales présentées sur les figures indiquent les écarts-types correspondants.

En ce qui concerne le nombre de feuilles émises par un point végétatif, indiquons que le nombre de feuilles considéré est celui qui est généré pàr le seul point végétatif marqué, sans tenir compte des feuilles émises sur d'éventuelles ramifications apparues à l'aisselle de ces feuilles au cours de la période d'observation. En situation de compétition pour la lumière, par analogie aux résultats observés sur graminées fourragères (Lemaire, 1985; Simon et Lemaire, 1987), les vitesses d'émission d'organes sont présentées en fonction des indices foliaires du couvert végétal.

\section{Résultats}

\section{Morphogénèse du trèfle blanc en l'absence de} compétition pour la lumière

\section{1) Emission des feuilles}

En phase d'implantation, la première feuille qui apparaît est unilobée; elle est déployée une dizaine de jours après la levée (Fig. 1). Les feuilles suivantes sont trilobées, leur vitesse d'émission étant pratiquement une fonction linéaire du temps: 0,18 feuille émise par jour dans les conditions de l'expérience. La légère rupture de pente observée sur la courbe vers le $26^{e}$ jour n'est pas en relation avec un changement du régime thermique, une présentation des résultats en fonction des sommes de températures n'améliorant pas la linéarité de la relation. 


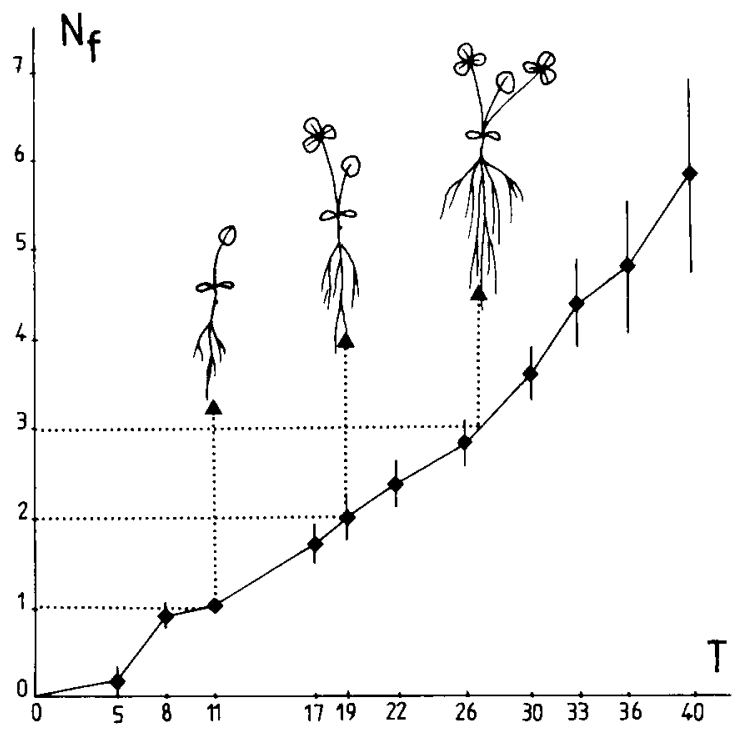

Fig. 1. Evolution du nombre de feuilles par plante (Nf) en fonction du temps $(T)$, exprimé en jours après la levée.

On peut indiquer que, pour la variété Huia, une nouvelle feuille trilobée est émise tous les $100^{\circ}$ j.

En couvert bien implanté, la dynamique d'apparition des feuilles présente 2 phases distinctes qui diffèrent selon le type de point végétatif (Fig. 2). Dans les 15 premiers jours qui suivent le baguage, la vitesse d'émission des feuilles (exprimée en nombre de feuilles émises par jour) est significativement plus importante sur les apex principaux $(0,269)$ que sur les apex secondaires $(0,217)$ et les points axillaires $(0,205)$. Plus tard, quand chaque type de point végétatif présente au moins 3 feuilles au-delà de la bague (soit 3 feuilles vraies pour les points axillaires), les vitesses d'émission des feuilles sont comparables pour les 3 types. Dans les conditions

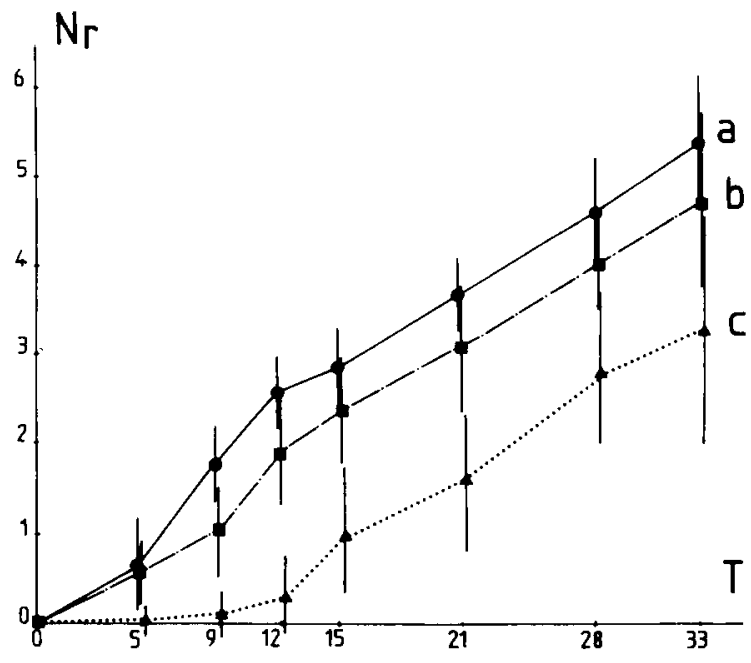

Fig. 2. Evolution du nombre de feuilles ( $\mathrm{Nf}$ ) en fonction du temps $(T)$ exprimé en jours après le baguage, pour les 3 types de points végétatifs $(a, b$ et $c)$ d'un couvert bien expérimentales rencontrées, ces vitesses sont respectivement de: 0,199 (type a), 0,191 (type b), 0,190 (type c).

Cette rupture de pente n'est pas liée à la température, une représentation graphique en fonction des sommes de températures ne changeant pas l'allure des courbes. Comme en phase d'implantation, on remarque que la variété Huia émet une nouvelle feuille tous les $100^{\circ} \mathrm{j}$.

\section{2) Emission des ramifications}

En phase d'implantation, une jeune plante de trèfle émet sa première ramification quand elle présente de 4 à 6 feuilles. En couvert bien implanté, une ramification apparaît sur les points végétatifs maintenus à la lumière, quand ces derniers présentent 3 à 4 feuilles développées à partir de la feuille axillante, et ce, quel que soit le type de point végétatif (Fig. 3).

La dynamique d'apparition de nouvelles ramifications sur les 3 types de points végétatifs considérés est présentée à la Figure 4. Pour les apex principaux et secondaires le nombre de nouvelles ramifications est une fonction linéaire du temps, les vitesses d'émission étant du même ordre de grandeur: 0,16 et 0,15 ramification apparue par jour, en moyenne sur la période. Pour les points axillaires, on observe une période de latence sans ramification, de 0 à 12 jours; ces points n'ayant pas encore atteint le stade critique de 3 feuilles pour ramifier. Au-delà, du $15^{\mathrm{e}}$ au $33^{\mathrm{e}}$ jour, la vitesse d'émission prend les mêmes valeurs que celles observées pour les 2 autres types: 0,142 (type a); 0,136 (type b); 0,135 (type c). Notons que les courbes présentées en fonc-

(a)

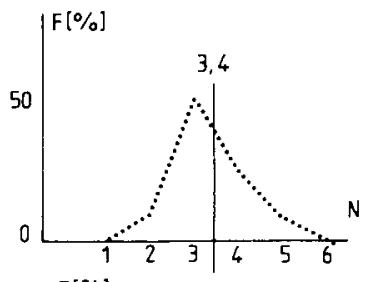

(b)

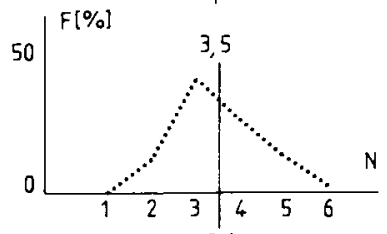

(C)

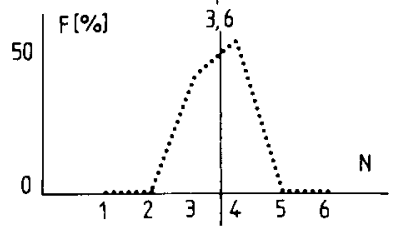

Fig. 3. Fréquence d'apparition de la première ramification $(F)$ en fonction du nombre de feuilles comptées à partir de la feuille axillante $(\mathrm{N})$, selon le type de point végétatif considéré ( $a$, b et $c)$. 


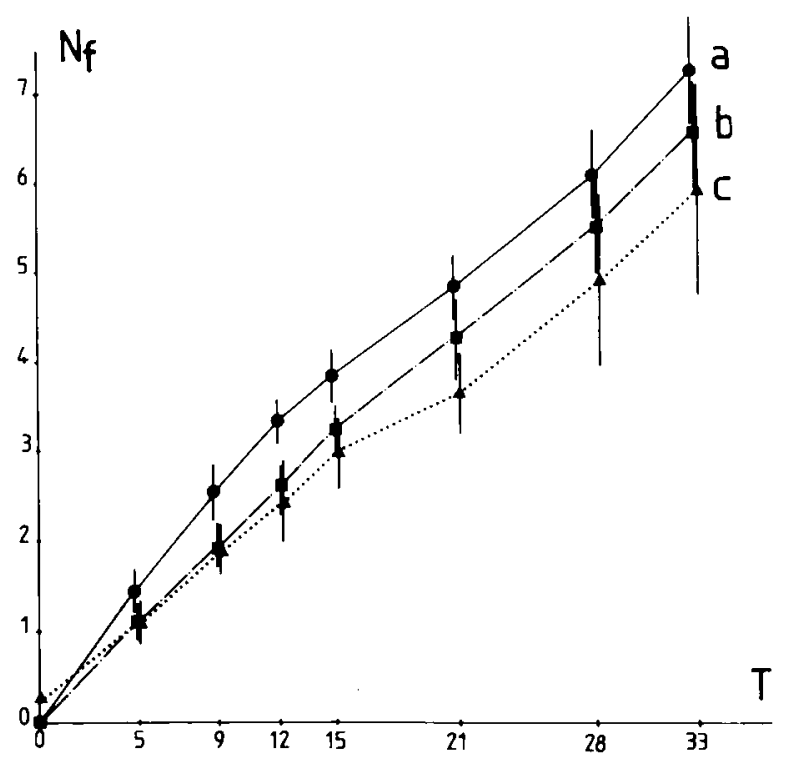

Fig. 4. Evolution du nombre de ramifications (Nr) en fonction du temps $(T)$, exprimé en jours après le baguage, pour les trois types de points végétatifs ( $a, b$ et $c)$.

tion des sommes thermiques ont la même allure, une ramification nouvelle étant émise tous les $100^{\circ}$ j pour cette variété, soit la même cadence d'émission que les feuilles.

Morphogénèse du trèfle blanc en conditions de compétition pour la lumière

\section{1) Emission des feuilles}

En couvert de trèfle blanc pur bien implanté, on constate que le rythme d'émission des feuilles des apex principaux situés au sein du couvert ne présente pas la même cinétique d'émission des feuilles que les apex comparables maintenus artificiellement à la lumière (Fig. 5a). Si la dynamique est comparable jusqu'au 19 e jour $(0,130$ contre 0,136 feuilles émises par jour), elle devient significativement plus faible par la suite $(0,062$ contre $0,117 \mathrm{f} / \mathrm{j}$, à partir du $26 \mathrm{e}$ jour $)$.

Si l'on exprime ces vitesses en fonction de l'indice foliaire du couvert (Fig. 5b), on remarque que celles-ci sont maximales pour les indices foliaires faibles, puis nettement ralenties au-delà de l'indice foliaire 3 , sans toutefois tendre vers 0 .

Ce phénomène n'est pas uniquement observé sur les apex principaux, mais aussi sur les autres types de points végétatifs. La vitesse d'émission des feuilles diminue significativement en fin de repousse, quel que soit le type de point végétatif considéré ( $\mathrm{a}, \mathrm{b}$ ou $\mathrm{c})$.

En phase d'implantation d'un couvert de trèfle blanc pur, ou d'un trèłle blanc associé à un raygrass anglais, les mêmes phénomènes ont été observés.

\section{2) Emission de ramifications}

Sur les mêmes points végétatifs que ceux qui ont servi à suivre l'évolution de l'apparition des
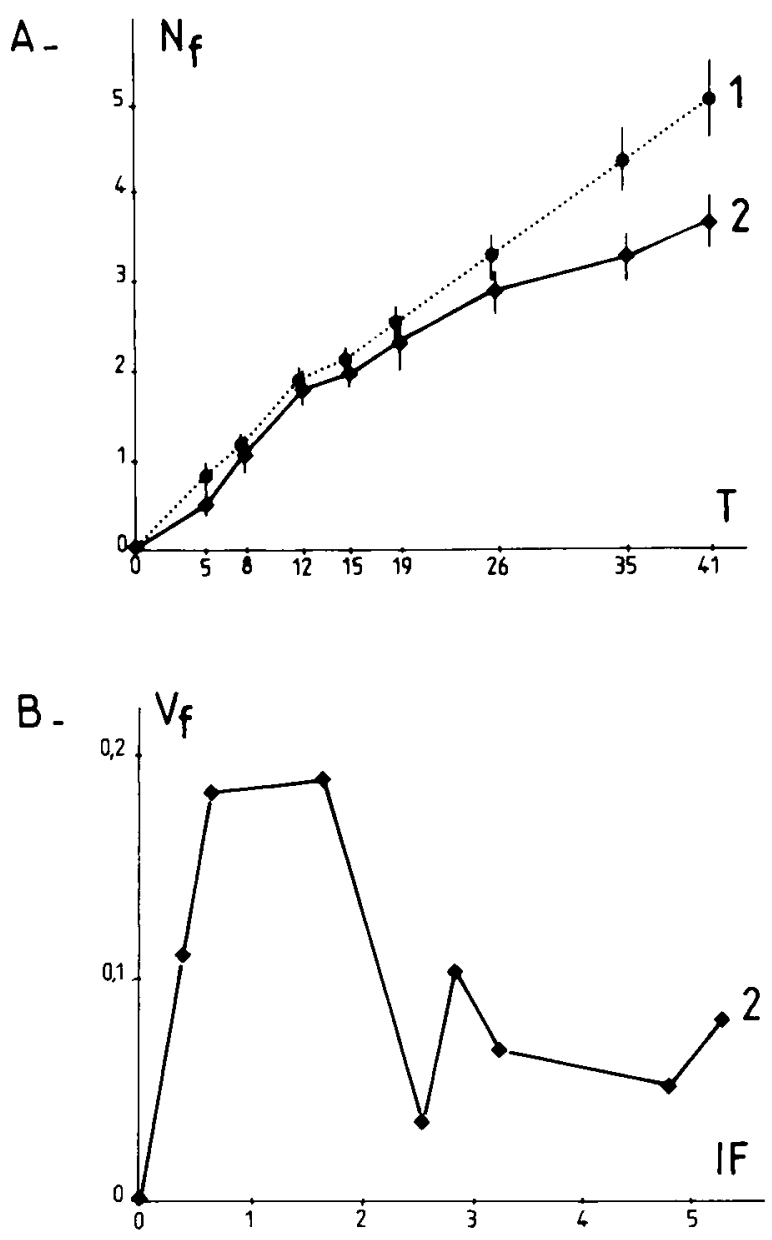

Fig. 5. A - Evolution en fonction du temps (T), exprimé en jours après le baguage, du nombre de feuilles (Aff) d'apex maintenus à la lumière (1) et d'apex situés dans un couvert en croissance (2); B - Evolution de la vitesse d'émission des feuilles (Vf) en fonction de l'indice foliaire (IF), dans ce dernier cas (2).

feuilles, nous avons observé l'émission des ramifications (Fig. 6).

Pour les apex principaux, le nombre de ramifications apparues par point végétatif bagué est non significativement différent de ceux maintenus à la lumière pendant les premiers jours de la repousse. Ensuite, la vitesse de ramification décroît par rapport au témoin à la lumière, puis s'annule après le $15^{\mathrm{e}}$ jour. Une dynamique semblable est observée pour les apex secondaires. En revanche, pour les points axillaires, la ramification est quasiment nulle au cours des 33 jours d'observation. Ces points atteignent difficilement le nombre critique de feuilles pour que commence la ramification quand l'indice foliaire est déjà égal à 3 . De ce fait, ils ne ramifient pas.

$\mathrm{Si}$ l'on exprime l'évolution des vitesses de ramification en fonction de l'indice foliaire, par analogie aux vitesses de tallage sur graminées fourragères (Simon et Lemaire, 1987), on remarque que la vitesse de ramification tend vers 
(a)

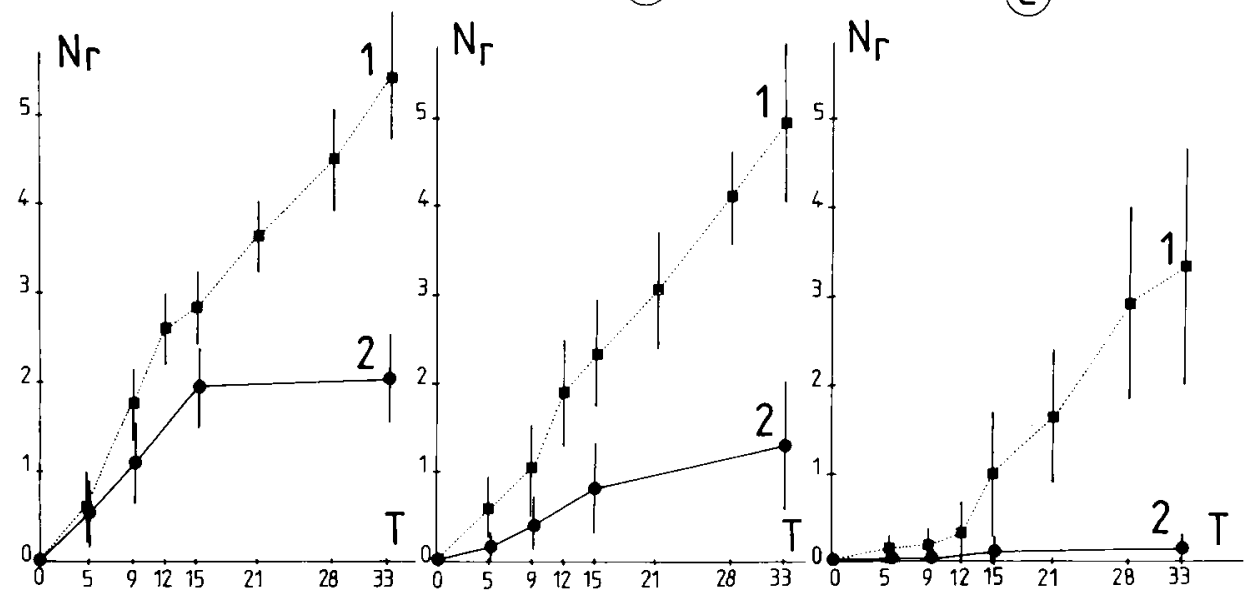

Fig. 6. Comparaison de l'évolution du nombre de ramifications (Nr) en fonction du temps (T) exprimé en jours après le baguage, par type de point végétatif ( $a$, b et $c$ ), entre situation à la lumière (1) et situation au sein du couvert (2).

0 dès que l'indice foliaire dépasse la valeur 3 (Fig. 7). Des observations analogues ont pu être faites en phase d'implantation d'une culture de trèfle blanc pur, ou d'une culture mixte avec raygrass anglais.

Les résultats obtenus à Lusignan, dans un autre contexte pédoclimatique, confirment ces cinétiques de ramification: vitesse d'émission des points végétatifs maximale en début de repousse et nulle en fin de repousse. L'interprétation est équivalente à celle développée sur les données de Quimper.

L'évolution du nombre de points végétatifs, mesuré par comptage sur des placettes élémentaires différant par leur niveau de fertilisation azotée (N0 et N1), est représentée à la Figure 8. Deux phases peuvent être distinguées:

- la première correspond à une situation où l'indice foliaire est faible (valeur inférieure à 3 ). Dans ce cas on observe une augmentation importante du nombre de points végétatifs par unité de surface;

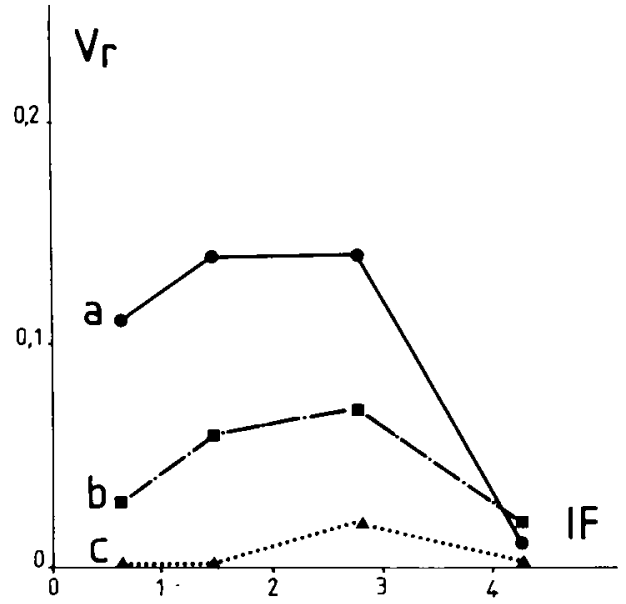

Fig. 7. Evolution de la vitesse de ramification $(\mathrm{Vr})$ en fonction de l'indice foliaire du couvert (IF), pour les trois types de points végétatifs $(a, b$ et $c)$.
- la seconde présente une décroissance très importante, qui correspond à une situation où:

- l'émission des points végétatifs est ralentie ou annulée, ce qui est en accord avec les résultats présentés plus haut pour les forts indices foliaires,

- il existe une mortalité importante d'apex. II n'est toutefois pas possible à partir de cette seule expérimentation de préciser si cette mortalité concerne plutôt les unités déjà en place à la coupe, où si elle touche plutôt les points végéta-
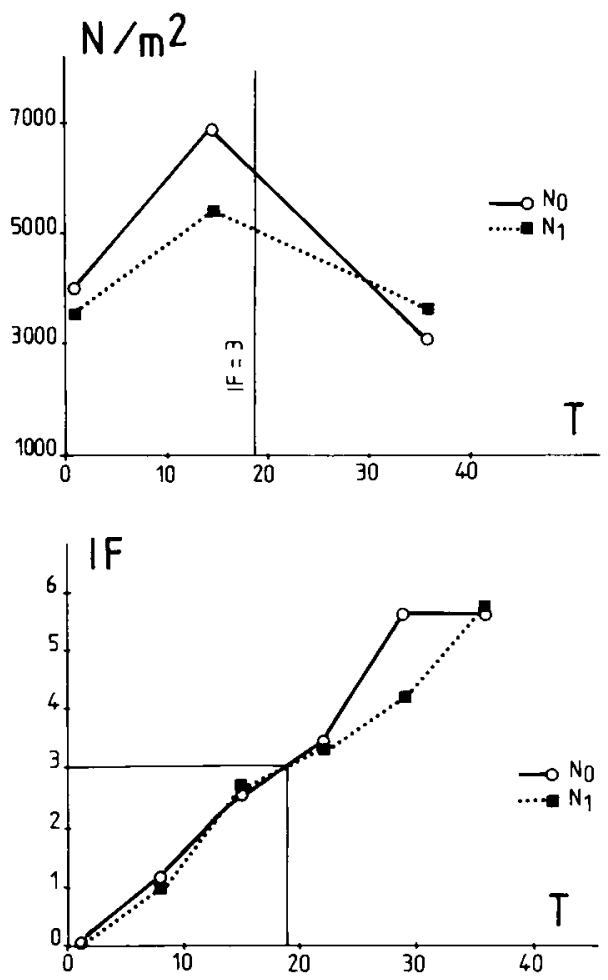

Fig. 8. Evolution du nombre $(\mathrm{N})$ de points végétatifs (tous types confondus) par unité de surface, en fonction du temps (T) exprimé en jour après la coupe, pour 2 niveaux de fertilisation azotée (No et N1) sur un trèfle blanc pur; évolution parallèle de l'indice foliaire (IF). 
tifs apparus lors de la première phase et qui sont globalement dominés par le couvert végétal.

\section{3) Allongement des entre-nœuds des apex prin- cipaux}

Sur l'essai conduit à Lusignan, la croissance en longueur des entre-nœuds des apex principaux a été suivie pendant la phase de repousse sur stolons bagués. Les résultats obtenus sur couvert de trèfle pur sont présentés à la Figure 9.

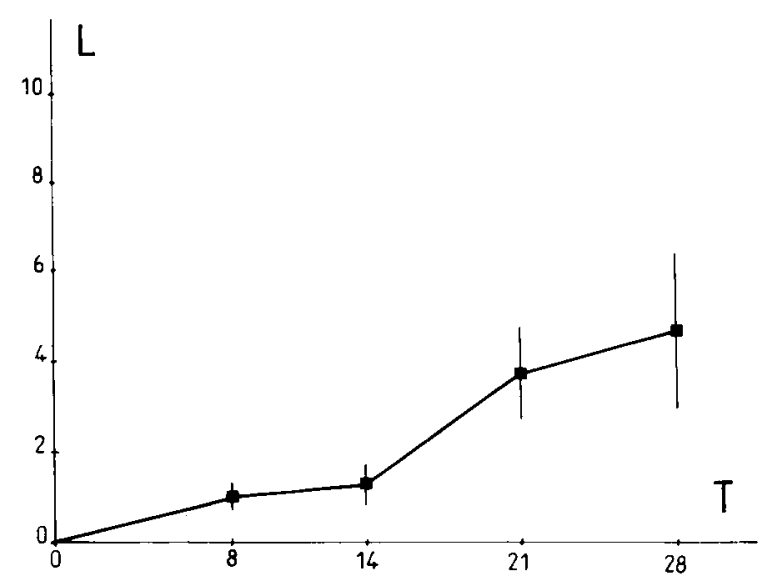

Fig. 9. Evolution des longueurs moyennes des entre-nœuds $(\mathrm{L}$ en $\mathrm{cm}$ ) des apex principaux, au cours d'une repousse de trèfle blanc, (T, temps exprimé en jours après la coupe).

Deux phases peuvent être distinguées sur le graphique : la première se caractérise par une vitesse d'allongement des entre-nœuds très réduite en début de repousse du 1 er au $15^{\mathrm{e}}$ jour, la seconde par une augmentation de la vitesse. II est important de souligner que le début de cette activité d'élongation est concommittante avec l'arrêt d'émission des points végétatifs.

\section{Discussion et conclusion}

Le comportement du trèfle blanc vis-à-vis du rythme d'émission des feuilles et des ramifications présente un certain nombre de similitudes avec les graminées fourragères tempérées.

En l'absence de compétition pour la lumière, on observe que:

- la vitesse d'émission des feuilles est sous la dépendance de la température. Elle peut être exprimée en fonction des sommes de températures, ce qui est en accord avec les observations de divers auteurs montrant la sensibilité du trèfle blanc à la température (par exemple Beinhart, 1963). Pour la variété Grasslands Huia observée ici, en prenant $0^{\circ} \mathrm{C}$ comme zéro de végétation, il faut $100^{\circ} \mathrm{j}$ pour qu'apparaisse une nouvelle feuille. Cependant, ce facteur n'est pas le seul à prendre en compte puisque l'on observe des dif- férences de vitesse entre les types de points végétatifs dans les jours suivants le baguage. On peut émettre l'hypothèse que des facteurs de nature trophique ou hormonale (dominance apicale) interviennent;

- la vitesse de ramification est corrélée à la vitesse d'émission des feuilles. Il s'agit d'une liaison de nature anatomique, puisque les points végétatifs apparaissent à l'aisselle des feuilles et qu'une seule ramification est portée par une feuille axillante;

- il y a un décalage de l'ordre de 3 phyllochrones entre l'émission d'une feuille et le développement du point végétatif placé à son aisselle, ce qui est également observé chez les graminées (Gounot et al., 1980; Franquin, 1974; etc.).

En situation de compétition pour la lumière, c'est-à-dire pour les indices foliaires supérieurs à 3-4 (un indice foliaire de 4 correspond à une extinction de $95 \%$ du rayonnement incident pour le trèfle blanc selon les mesures que nous avons effectuées, résultats qui confirment ceux de Brown et Blaser (1968)), on observe que:

- le rythme d'émission des feuilles est significativement ralenti. Brougham (1958) avait remarqué que l'émission des feuilles était maximale au cours des 20 premiers jours suivant une coupe, au-delà, elle décroissait sensiblement;

- le rythme d'émission des points végétatifs est nettement plus affecté, puisqu'il s'annule pour des indices foliaires voisins de 3-4.

Là aussi, l'analogie est conservée par rapport au rythme d'émission des talles des graminées fourragères, pour lesquelles le tallage est stoppé dans les couverts ou pour qui l'indice foliaire dépasse la valeur 3-4 (Simon et Lemaire, 1987).

Concernant la nature des mécanismes impliqués dans ces effets de la compétition pour la lumière, 2 hypothèses peuvent être avancées. L'une fait appel à une limitation de la disponibilité en substrats carbonés; cette hypothèse a souvent été invoquée pour expliquer l'arrêt du tallage sur graminée (Masle-Meynard, 1980). Une seconde hypothèse peut être envisagée, faisant appel aux réactions de la morphogénèse à la composition spectrale du rayonnement. Sur graminée il est démontré qu'une diminution du rapport rouge proche / rouge lointain a pour conséquence une nette diminution de la vitesse de tallage (Deregibus et al., 1985). Par ailleurs, l'augmentation de la compétition dans un couvert entraîne une diminution de ce rapport (Holmes, 1981). De plus, il apparaît que la morphogénèse du trèfle blanc est également sensible à la composition spectrale du rayonnement. Une modification de cette dernière, simulant un phytoclimat d'ombrage, entraîne une diminution de la ramifi- 
cation par rapport au témoin recevant le même éclairement photosynthétiquement actif (Solangaarachi et Harper, 1987), ce qui est en accord avec nos observations.

Enfin, la seule hypothèse d'une limitation trophique ne peut expliquer pourquoi, lorsque la compétition pour la lumière devient importante, nous observons sur les mêmes stolons une diminution de l'émission de points végétatifs et une stimulation de la vitesse d'élongation des entrenœuds. De même, cette unique hypothèse ne peut expliquer les résultats de Robson et Poliaszek (1984). Dans ces travaux, des sections de stolons comportant chacune deux ramifications sont cultivées soit en pleine lumière, soit sous ombrage, soit l'une en pleine lumière et l'autre sous ombrage. Dans cette dernière configuration, une partie du carbone assimilé par les feuilles recevant un éclairement fort est transféré à la ramification ombrée. Ce supplément de carbone conduit à une stimulation de l'élongation des entre-nœuds du stolon ombré dont les feuilles conservent une morphologie étiolée. Cet ensemble de résultats suggère que la plante intègre un autre mode de perception de la compétition pour la lumière que le simple aspect quantitatif déterminant la disponibilité en carbone.

En conséquence, il nous semble que les réactions du trèfle blanc à la compétition pour la lumière intègrent à la fois des phénomènes de nature photomorphogénétique et des phénomènes de nature trophique. $\mathrm{Ce}$ dernier point pourrait expliquer les disparitions de points végétatifs observées en fin de cycle de croissance.

\section{Références}

Beinhart G. (1963) Effect of environment on meristematic development, leaf area, and growth of white clover. Crop Sci. 3, 209-213

Bonhomme R. \& Variet-Grancher C. (1977) Application aux couverts végétaux des lois de rayonnement en milieu diffusant. I - Etablissement des lois et vérifications expérimentales. Ann. Agron. 28, 6, 567-582

Bonhomme R. \& Varlet-Grancher C. (1979) Application aux couverts végétaux des lois de rayonnement en milieu diffusant. II - Interception du rayonnement solaire par une culture. Ann. Agron. 30, 1, 1-26
Brougham R.W. (1958) Leaf development of a pure stand of white clover after defoliation. Aust. J. Agric. Res. 9, 39-52

Brown R.H. \& Blaser R.E. (1958) Leaf area index in pasture growth. Herb. Abstr. 38, 1, 1-9

Deregibus V.A., Casal J.J. \& Sachez R.A. (1985) Variations in tiller dynamics and morphology in Lolium multiflorum Lam. Vegetative and reproductive plants as affected by differences in red / far-red irradiation. Ann. Bot. 56, 553-559

Franquin P. (1974) Développement de la structure fondamentale ou développement morphogénétique de la plante. Cah. ORSTOM, Sér. Biol. 23, 23-30

Gallagher J.N. \& Biscoe B.V. (1978) Radiation absorption growth and yield of cereals. J. Agric. Sci. Camberra $91,47-60$

Gosse G., Chartier M. \& Lemaire G. (1984) Mise au point d'un modèle de prévision de production pour une culture de luzerne. C. R. Acad. Sci. Paris 298, Sér. 3, $18,541-544$

Gounot M., Atry M., N'Kandza J. \& Yu O. (1980) Photosynthèse nette et rythme d'apparition des feuilles chez le dactyle (Dactylis glomerata L.), C. R. Acad. Sci. Paris Sér. D. 290, 1257-1260

Holmes M.G. (1981) Spectral distribution of radiation within plant canopies. In : Plants and daylight spectrum. Proceedings of the First International Symposium of the British Photo-biology Society. Academic Press. pp. 147-158

Lemaire G. (1985) Cinétique de croissance d'un peuplement de fétuque élevée pendant l'hiver et le printemps. Thèse de doctorat d'Etat, Université de Caen

Masle-Meynard J. (1980) L'élaboration du nombre d'épis chez le blé d'hiver. Influence de la structure du peuplement sur l'utilisation de l'azote et de la lumière. Thèse de docteur-ingénieur, INA Paris-Grignon

Monteith J.L. (1977) Climate and the efficiency of crop production in Britain. Phil. Trans. R. Soc., London B $281,277-297$

Robson M.J. \& Poliaszek T. (1984) Effect of contrasting light regime on the growth and morphology of individual plants of white clover and of different stolons on the same plant. In: Forage Legumes. (D.J. Thompson, British Grassland Society Occasional Symposium, 16, 189-190 ed.)

Simon J-C. \& Lemaire G. (1987) Tillering and leaf area index in grasses in the vegetative phase. Grass Forage Sci. 42, 373-380

Solangaarachchi S.M. \& Harper J.L. (1987) The effect of canopy filtered light on the growth of white clover (Trifolium repens). Oecologia 72, 372-376

Varlet-Grancher C. \& Bonhomme R. (1982) Efficience de la conversion de l'énergie solaire par un couvert végétal. Acta Oecol. Oecol. Plant. 17, 1, 3-26 